\title{
Evolución de los glaciares de los nevados Collquepucre, Llongote, Pariacaca y Ticcla en la cuenca del río Cañete
}

\author{
Miguel Alva Huayaney \\ Universidad Nacional Mayor de San Marcos \\ <malvah@unmsm.edu.pe > \\ Robert Ramos Alonzo \\ Universidad Católica Sedes Sapientiae \\ < rramosa@ucss.edu.pe>
}

\section{RESUMEN}

En la cuenca del río Cañete, se observa un proceso de retroceso glaciar constante desde hace más de 37 años. Basado en fotografías aéreas para los años 1962 y 1970, y utilizando imágenes Landsat del periodo 1990-2015, se ha podido establecer que la superficie glaciar de los nevados Collquepucre, Llongote, Pariacaca y Ticcla ha disminuido en proporción de 2 a $7 \mathrm{~km}^{2}$, por cada 5 años; situación que guarda relación con la dinámica de los glaciares tropicales en donde diversas fuentes indican que son los ecosistemas más frágiles ante el cambio climático.

PALABRAS Clave: nevado; glaciares; volumen glaciar; retroceso glaciar; cambio climático.

\section{Evolution of the Collquepucre, Llongote, Pariacaca and Ticcla glaciers in the Cañete river basin}

\section{ABSTRACT}

In the Cañete River Basin, a glacial retreat has been observed for more than 37 years. Based on aerial photographs for the years 1962 and 1970, and using Landsat images from the 1990 - 2015 period, the glacier surface of the Collquepucre, Llongote, Pariacaca and Ticcla snowfalls has decreased by 2 to $7 \mathrm{~km}^{2}$, for every 5 years; a situation that is related to the dynamics of tropical glaciers where various sources indicate that they are the most fragile ecosystems in the face of climate change.

KeYworDs: Glacier-covered area; Glacier ice volume; Glacier's higher of line equilibrium; Glacier's accumulation area. 


\section{Introducción}

A proximadamente el $99 \%$ de los glaciares tropicales del mundo se encuentran distribuidos en los Andes de Sudamérica, de los cuales el $71 \%$ se localizan sobre las cordilleras del Perú (G. Kaser y H. Georges, 1997). En los glaciares de la cuenca alta del río Cañete, como se aprecia en la actualidad, solo existen once nevados de los dieciséis existentes hace 37 años (fotografías aéreas 1962), con lo cual se confirma un acelerado proceso de desglaciación en la zona; teniéndose para el caso de los nevados Huaynacutuni, Shicra, Tunsho, Tapo y Escalera un proceso completo de desglaciación $(100 \%)$.

En la zona de la cuenca del río Cañete se encuentran tanto glaciares como glaciaretes, si bien se les tiende a considerar indistintamente como "glaciares». Vale la pena hacer la distinción conceptual entre los dos tipos dadas las importantes diferencias en sus características, dimensiones y propiedades dinámicas. Un glaciar es «toda masa de hielo perenne, formada por acumulación de nieve, cualesquiera sean sus dimensiones y formas, el que fluye lentamente por deformación, deslizamiento basal y deslizamiento de sedimentos subglaciales». Lliboutry (1956, citado por IGP 2009). Una de las principales propiedades que definen a un glaciar como tal es precisamente la evidencia de flujo de hielo en el presente o bien en el pasado (Cogley et al., 2011). Por su parte, un glaciarete es un glaciar muy pequeńo, que perdura por al menos dos años consecutivos, típicamente menos de $0.25 \mathrm{~km}^{2}$ (25 ha) de superficie, que no exhibe evidencia marcada de flujo en superficie (Cogley et al., 2011).

En general, la disminución del área glaciar en la cuenca alta del río Cañete en un promedio de tiempo de 37 años es de $85.20 \%$, cifra por supuesto muy elevada. Por consiguiente, se estima que de seguir este fenómeno de deglaciación, en un periodo muy cercano, 10 a 15 ańos, la superficie glaciar será mínima.

Otro aspecto de resaltar es la importancia de la cuenca alta, denominada en el presente estudio como subcuenca Tanta, debido a que en esta se ubica el 94\% del área glaciar, posibilitando la existencia de catorce lagunas, consideradas dentro de la cuenca como las más importantes; ambas fuentes originan el nacimiento del río Cañete.

Con el objetivo de obtener una estimación de la desglaciación de los nevados de la cuenca del río Cañete, se realizó un análisis de regresión lineal simple con los parámetros de año y área de la cobertura glaciar, para lo cual se usaron programas de tratamiento de imágenes satelitales Erdas y Sistemas de Información Geográfica ArcGIS. La correlación es buena con un $\mathrm{R} 2=0.93$ resultaron estadísticamente significativas.

\section{2. Área de estudio}

Los nevados que comprenden la presente investigación se ubican en las partes más altas de la cuenca del río Cañete, esta cuenca forma parte de las provincias de Cañete y Yauyos, pertenecientes ambas al departa-

\section{FIGURA 1: UBICACIÓN DE LA CUENCA DEL RÍ0 CAÑETE}

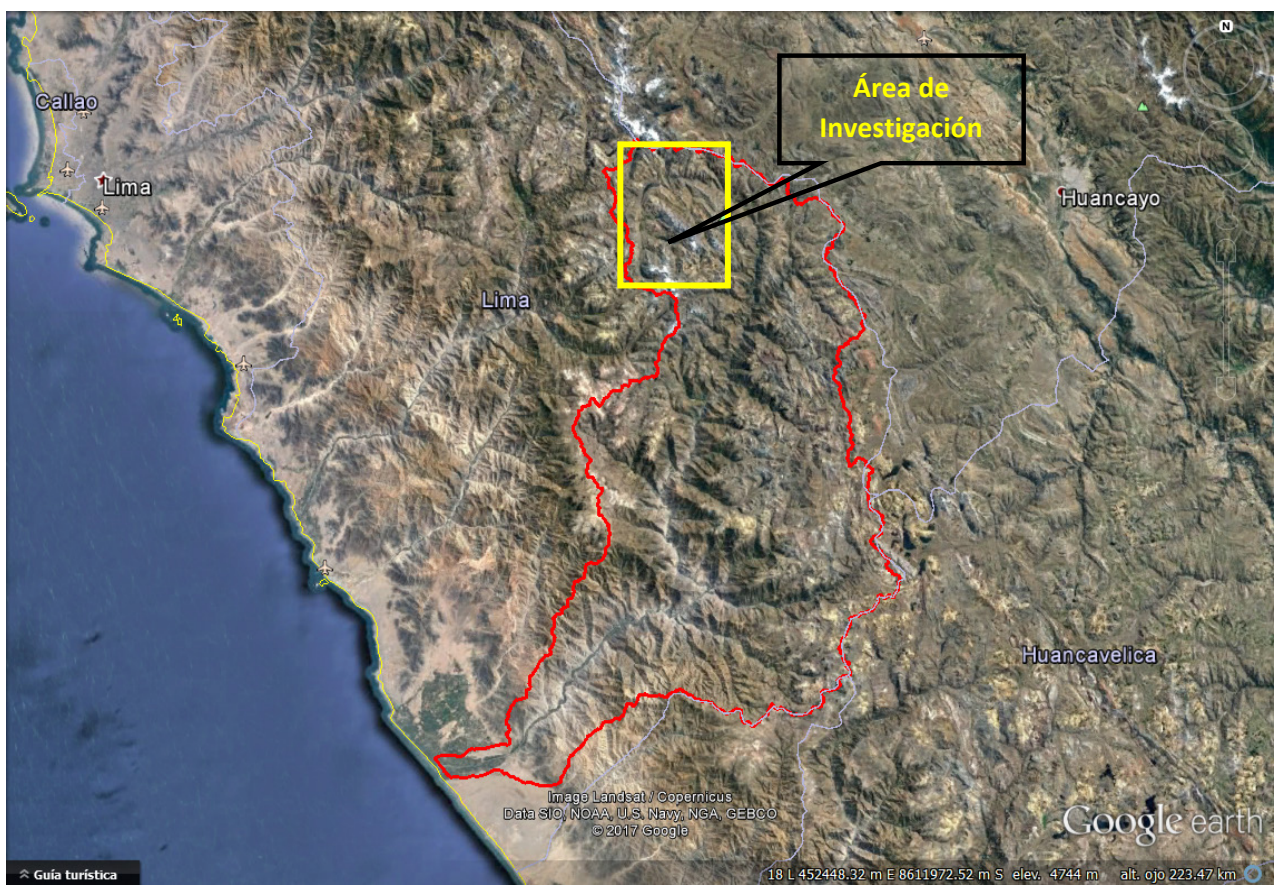

Fuente: Google Earth (2017), límite de cuencas ANA. 
mento de Lima. Geográficamente, se encuentra entre

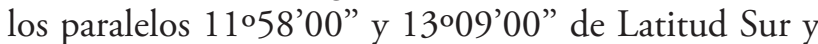
los meridianos $75^{\circ} 31^{\prime} 00^{\prime \prime}$ y $76^{\circ} 31^{\prime} 00^{\prime \prime}$ de Longitud Oeste.

La cuenca del río Cañete tiene una extensión aproximada de $6192 \mathrm{~km}^{2}$, de los cuales el 78,4\% $\left(4856 \mathrm{~km}^{2}\right)$ corresponde a la cuenca húmeda. El río Cañete nace en la laguna Ticllacocha, ubicada al pie de las cordilleras de Ticlla y Pichahuarco, en la divisoria de cuencas con el río Mala. Sus recursos hídricos provienen de los aportes de la lluvia, así como los derivados de lagunas y deshielo de los nevados, ubicados estos principalmente en el extremo norte de la cuenca y sobre los $4500 \mathrm{~m}$.

\section{Objetivo}

\subsection{General}

Determinar la dinámica del área glaciar de los nevados Collquepucre, Llongote, Pariacaca y Ticcla usando imágenes satelitales del periodo 1990 al 2015.

\subsection{Especificos}

- Determinar el volumen de los nevados Collquepucre, Llongote, Pariacaca y Ticcla usando la formula empírica de Bahr y Klein Isacks.

- Determinar la Línea de Equilibrio Glaciar en función de la temperatura y de la precipitación media anual.

- Determinar el Área de Acumulación Glaciar.

\section{Metodología}

Se ha considerado la siguiente metodología.

- Descargar imágenes satelitales Lansat 5 ETM+ y Landsat 8 OLI a partir del servidor ESDI de Estados Unidos (http://glcfapp.glcf.umd.edu:8080/esdi/).

- Se realizó el tratamiento de la imagen satelital con ayuda del programa Erdas Imagine para integrar las bandas espectrales en un solo archivo.

- Se halló el Índice de Nieve de Diferencia Normalizada (NDSI) con las bandas 2 (verde) y la banda 5 (infrarrojo medio). (https://ntrs.nasa.gov/ archive/nasa/casi.ntrs.nasa.gov/20100031195. pdf).
- Se trazó Calculo de la Línea de Equilibrio Glaciar (ELA).

- Se determinó el área glaciar y los respectivos volúmenes de los nevados Collquepucre, Llongote, Pariacaca y Ticcla.

\section{Resultados}

\subsection{Determinación del área de los glaciares}

Las áreas de los nevados fueron obtenidas mediante el uso de un método de clasificación no supervisada, a partir de imágenes LANDSAT 5 TM LANDSAT 8 OLI, con lo que a partir de ellos se ha realizado la combinación de las bandas para poder diferenciar el glaciar respecto a otros elementos del relieve.

A partir de las imágenes correspondientes a los años 1990, 1995, 2000, 2005, 2010 y 2015, se calcularon las áreas de los nevados Ticlia, Llongote (al sur del área de estudio) y los nevados Pariacaca y Collquepucro (al norte), de este modo se obtuvieron los siguientes datos.

\section{FIGURA 2: UBICACIÓN DE LOS NEVADOS}

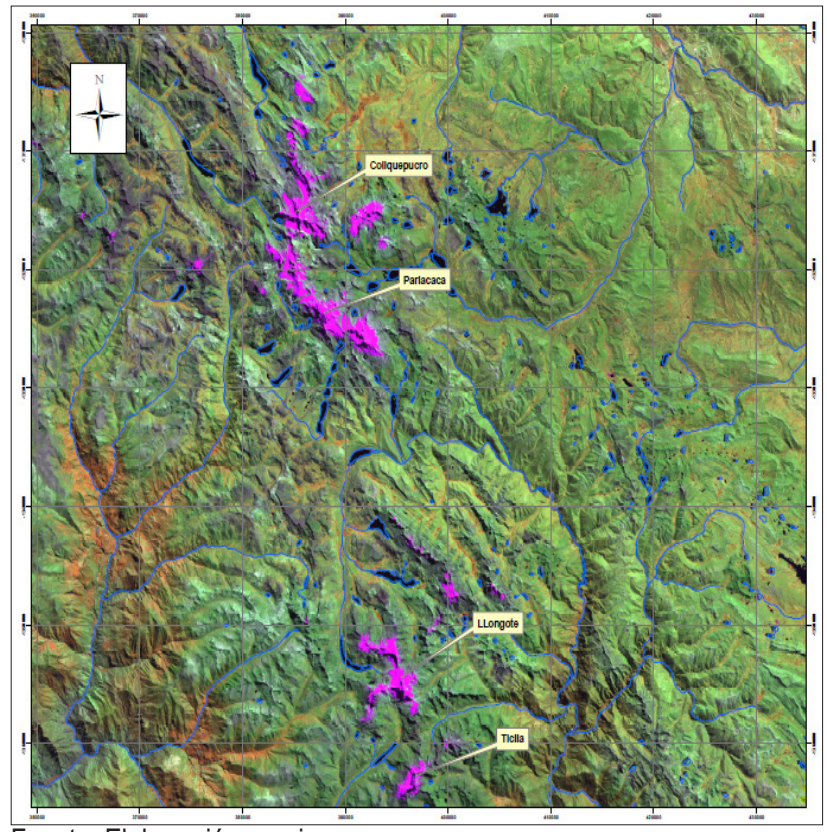

Fuente: Elaboración propia.

En 1990 el área de los nevados analizados fue de $68.9 \mathrm{~km}^{2}$, para el ańo 1995 presentó un área de 62.1 $\mathrm{km}^{2}$, en tanto que para el año 2000 su área fue de 57.34 $\mathrm{km}^{2}$, en el $2005 \mathrm{el}$ área fue de $54.75 \mathrm{~km}^{2}$, en el 2010 el área era de $52.98 \mathrm{~km}^{2}$ y en el 2015 el área fue de 
FIGURA 3: NEVADOS EN EL SECTOR NORTE, QUE SIRVEN DE LÍMITE DE CUENCA DEL RÍO CAÑETE

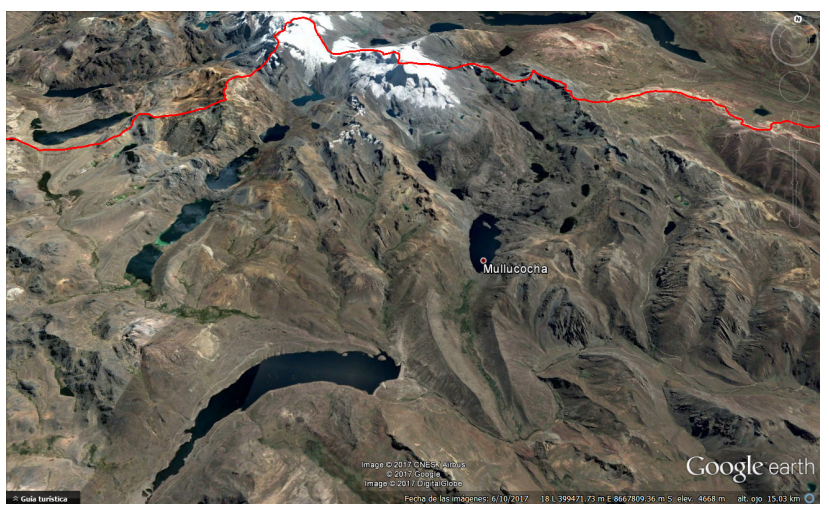

Fuente: Google Earth

FIGURA 4: NEVADOS EN EL SECTOR SUR, QUE SIRVEN DE LÍMITE DE CUENCA DEL RÍO CAÑETE

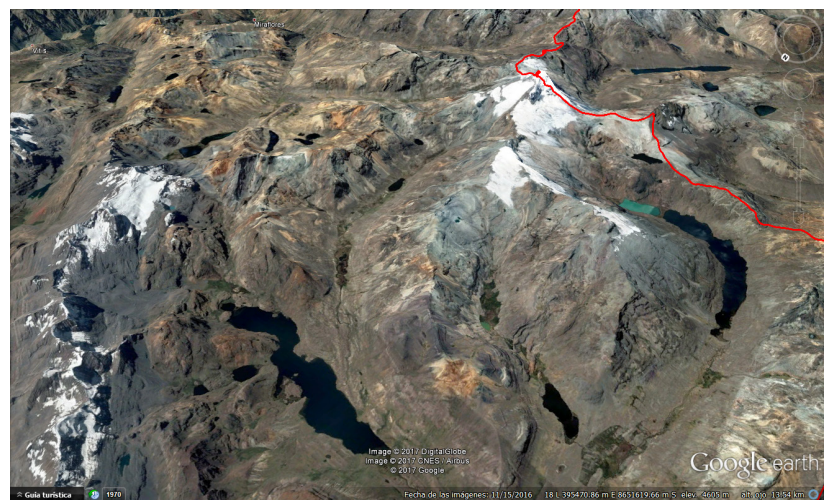

Fuente: Google Earth

$47.74 \mathrm{~km}^{2}$; en este periodo (1990-2015) los nevados presentaron una pérdida del $30 \%$ de su área respecto año cero (1990). El detalle de lo descrito se presenta en el Cuadro 1.

\section{CUADRO 1: ÁREA DE LOS NEVADOS COLLQUEPUCRE,} LLONGOTE, PARIACACA Y TICCLA, SEGÚN AÑOS

\begin{tabular}{|c|c|c|c|}
\hline Años & Área $\left(\mathrm{Km}^{2}\right)$ & Pérdida $\left(\mathrm{km}^{2}\right)$ & Pérdida $(\%)$ \\
\hline 1990 & 68.90 & & \\
\hline 1995 & 62.10 & 6.80 & 9.9 \\
\hline 2000 & 57.34 & 11.56 & 16.8 \\
\hline 2005 & 54.75 & 14.15 & 20.5 \\
\hline 2010 & 52.98 & 15.92 & 23.1 \\
\hline 2015 & 47.74 & 21.16 & 30.7 \\
\hline \multicolumn{2}{|c|}{ Pérdida total } & 21.16 & 30.7 \\
\hline
\end{tabular}

Fuente: Elaboración propia

\subsection{Cálculo de la desaparición de los nevados}

Para determinar el año aproximado en que podrían desaparecer los nevados, se sigue el método de la regresión lineal simple. Primero se definen las variables X (área) e Y (año), una de ellas depende directamente de la otra; así la disminución del área de los nevados es dependiente de los años. Dada la dependencia de una variable respecto a otra se considera la aplicación de una función lineal el que se define como: $\mathrm{Y}=\mathrm{a}+\mathrm{Xb}$ (ecuación de la línea recta), donde «b» es la pendiente (inclinación) y «a» es el intercepto, es decir, el valor donde la recta corta o intercepta al eje Y.

Fórmula para hallar el valor b:

$$
B=\frac{\sum X Y-n \bar{X} \bar{Y}}{\sum X^{2}-n \bar{X}^{2}}
$$

Fórmula para determinar a:

\section{$\mathrm{a}=\bar{Y} \overline{-b} \bar{X}$}

Donde:

$\bar{X}=$ Promedio de los valores de $\mathrm{X}$

$\bar{Y}=$ Promedio de los valores de $\mathrm{Y}$

$\Sigma \mathrm{Y}^{2}=$ Sumatoria de los cuadrados de los valores de $\mathrm{Y}$

$\Sigma \mathrm{X}^{2}=$ Sumatoria de los cuadrados de los valores de $\mathrm{X}$

$\boldsymbol{\Sigma} \mathbf{X Y}=$ Sumatoria entre los productos entre $\mathrm{X}$ e $\mathrm{Y}$

Con esta consideración se procedió a calcular el área 0 , es decir, el año en que podrían desaparecer los nevados Ticlia, Llongote, Pariacaca, Collquepucro (análisis conjunto).

CUADRO 2: CÁLCULO DE R

\begin{tabular}{|l|l|}
\hline \multicolumn{2}{|c|}{ Estadísticas de la regresión } \\
\hline Coeficiente de correlación múltiple & 0.966 \\
\hline Coeficiente de determinación $\mathrm{R}^{\wedge} 2$ & 0.934 \\
\hline $\mathrm{R}^{\wedge} 2$ ajustado & 0.912 \\
\hline Error típico & 1.903 \\
\hline
\end{tabular}

Fuente: Elaboración propia 
FIGURA 5: FIGURAS COMPARATIVAS DEL RETROCESO GLACIAR EN LA CUENCA DEL RÍO CAÑETE
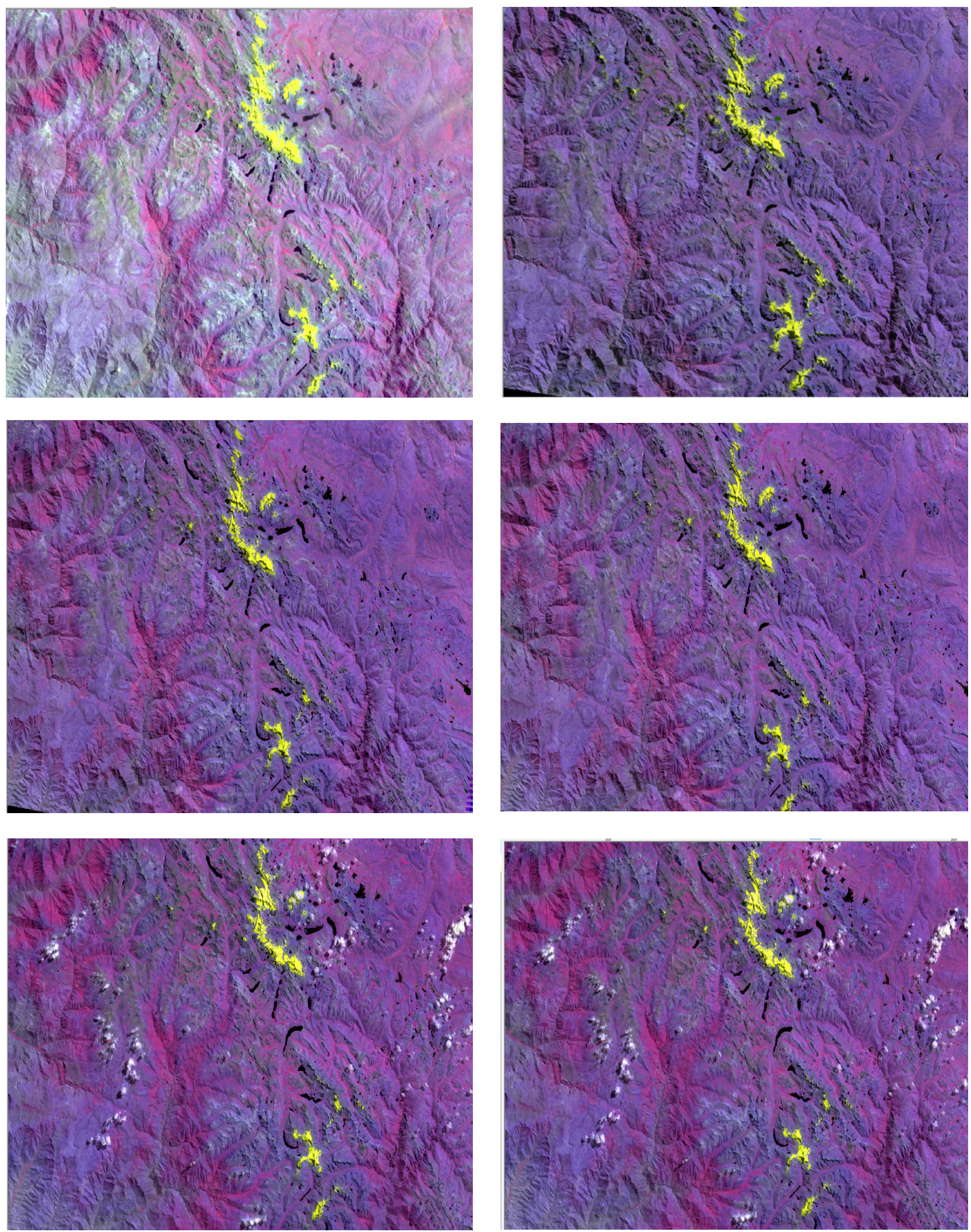

Fuente: Elaboración propia 
FIGURA 6: RECTA DE REGRESIÓN LINEAL, APLICADO PARA DETERMINAR EL AÑO EN QUE DESAPARECERÍA LOS NEVADOS

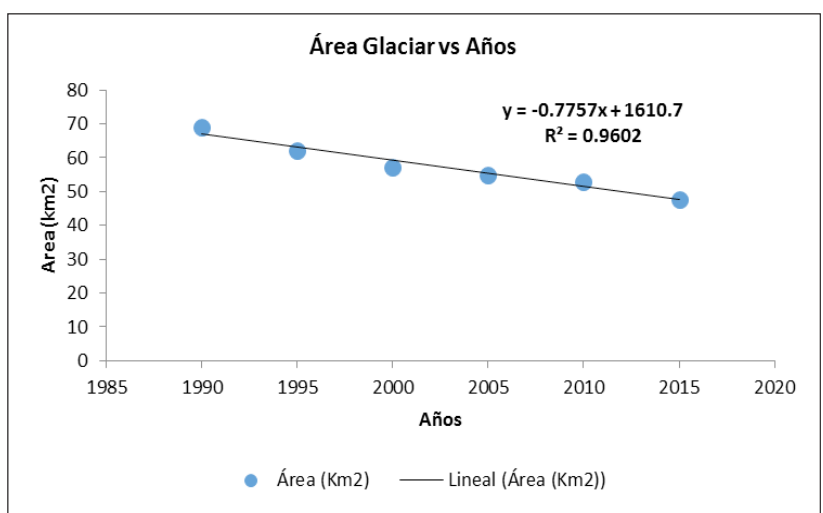

Fuente: Elaboración propia

El resultado del $\mathrm{r}$ está por encima de 0.9, esto indica que hay una buena correlación entre los datos de ingreso (disminución de las áreas de los nevados respecto a los ańos).
CUADRO 4: CALCULO DEL ÁREA DE LOS NEVADOS EN QUE SU ÁREA SEA $0 \mathrm{KM}^{2}$

\begin{tabular}{|c|c|}
\hline Años & Área $\left(\mathrm{km}^{2}\right)$ \\
\hline 1990 & 68.90 \\
\hline 1995 & 62.10 \\
\hline 2000 & 57.34 \\
\hline 2005 & 54.75 \\
\hline 2010 & 52.98 \\
\hline 2015 & 47.74 \\
\hline $2076^{*}$ & 0.00 \\
\hline
\end{tabular}

$\left(^{*}\right)$ : Año aproximado donde podrían desaparecer los nevados Fuente: Elaboración propia.

\subsection{Cálculo del volumen del glaciar}

Para calcular el volumen glaciar se sigue la metodología utilizada de Bahr et ál. (1996, 1997) y Klein Isacks (1999).

CUADRO 3: CÁLCULOS ESTADÍSTICOS

\begin{tabular}{|l|c|c|c|c|c|c|c|c|}
\hline & Coeficientes & $\begin{array}{c}\text { Error } \\
\text { típico }\end{array}$ & Estadístico t & Probabilidad & $\begin{array}{c}\text { Inferior } \\
95 \%\end{array}$ & $\begin{array}{c}\text { Superior } \\
95 \%\end{array}$ & $\begin{array}{c}\text { Inferior } \\
95.0 \%\end{array}$ & $\begin{array}{c}\text { Superior } \\
95.0 \%\end{array}$ \\
\hline Intercepción & 1627 & 240.7 & 6.758 & 0.007 & 860.8 & 2393 & 860.8 & 2393 \\
\hline Variable X1 & -0.78 & 0.12 & -6.51 & 0.007 & -1.17 & -0.4 & -1.17 & -0.4 \\
\hline
\end{tabular}

Fuente: Elaboración propia

El cambio climático manifestado en una distorsión de las características climáticas en diferentes partes del mundo está generando graves consecuencias en los recursos hídricos, estos cambios son básicamente el incremento gradual de la temperatura, el que se está incrementando dramáticamente en estos últimos años. Así los nevados se constituyen en los más sensibles a los cambios ante el incremento de la temperatura.

Para el análisis del retroceso glaciar se han considerado imágenes desde 1990 hasta el 2015, con un rango de análisis de 5 años, estableciéndose que entre 1990 y 1995 el glaciar ha perdido un área de $6.8 \mathrm{~km}^{2}$, entre 1995 y 2000 un área de $4.76 \mathrm{~km}^{2}$, entre el 2000 y 2005 un área de $2.59 \mathrm{~km}^{2}$, entre el 2005 y 2010 un área de $1.77 \mathrm{~km}^{2}$, y entre el 2010 y 2015 un área de $5.24 \mathrm{~km}^{2}$. Bajo esta consideración se estima que el glaciar ira perdiendo cada vez su área glaciar hasta que en la década del 2070 podría desaparecer por completo.

\section{Fórmula propuesta}

$$
\mathrm{V}=\mathrm{CA}^{\mathrm{b}}
$$

Donde $\boldsymbol{A}$ es el área del glaciar en $\mathrm{km}^{2} ; \boldsymbol{V}$ volumen de hielo en $\mathrm{km}^{3} ; \boldsymbol{c}$ un parámetro lineal $(\mathrm{c}=0.048)$ y $\boldsymbol{b}$ un factor exponencial $(b=1.36)$.

Siguiendo este procedimiento se ha realizado una aproximación del volumen total de los nevados estudiados. Así en 1990 superaba los $15 \mathrm{~km}^{3}$, disminuyendo $2.0 \mathrm{~km}^{3}$ para el año 1995 , mientras que en ese año se estima que el volumen estaba en $13.18 \mathrm{~km}^{3}$. Este volumen disminuyó hasta $11.82 \mathrm{~km}^{3}$ (pérdida total de $3.36 \mathrm{~km}^{3}$ ), en el año $2000 \mathrm{el}$ volumen se encontraba en $11.82 \mathrm{~km}^{3}$ y para el 2005 el volumen se encontraba en $11.1 \mathrm{~km}^{3}$ (con una pérdida de 4.08 $\mathrm{km}^{3}$, en el 2010 el volumen fue de $10.62 \mathrm{~km}^{3}$, lo que significó una pérdida de $4.56 \mathrm{~km}^{3}$ finalmente para el 2015 el volumen era de $9.22 \mathrm{~km}^{3}$ (pérdida de 5.96 $\mathrm{km}^{3}$ ). como es de evidenciar es claro la pérdida del 
volumen glaciar respecto a años los anteriores, por lo que la pérdida del volumen glaciar desde 1990 hasta el 2015 fue del $30 \%$.

CUADRO 5: VOLUMEN Y PÉRDIDA DE VOLUMEN DE LOS NEVADOS ESTUDIADOS

\begin{tabular}{|c|l|c|c|c|}
\hline Año & Cálculo & $\begin{array}{c}\text { Volumen } \\
\left(\mathrm{km}^{3}\right)\end{array}$ & $\begin{array}{c}\text { Pérdida } \\
\left(\mathrm{km}^{3}\right)\end{array}$ & $\begin{array}{c}\text { Pérdida } \\
(\%)\end{array}$ \\
\hline 1990 & $0.048^{\star}(68.90)^{1.36}$ & 15.18 & & \\
\hline 1995 & $0.048^{\star}(62.10)^{1.36}$ & 13.18 & 2 & 13.2 \\
\hline 2000 & $0.048^{\star}(57.34)^{1.36}$ & 11.82 & 3.36 & 22.1 \\
\hline 2005 & $0.048^{\star}(54.75)^{1.36}$ & 11.1 & 4.08 & 26.9 \\
\hline 2010 & $0.048^{\star}(52.98)^{1.36}$ & 10.62 & 4.56 & 30.0 \\
\hline 2015 & $0.048^{\star}(47.74)^{1.36}$ & 9.22 & 5.96 & 39.3 \\
\hline Pérdida total & & & 5.96 & 39.3 \\
\hline
\end{tabular}

Fuente: Elaboración propia

\subsection{Cálculo de la línea de equilibrio glaciar}

La Línea de Equilibrio Glaciar (ELA) es la línea que separa la zona de acumulación de la zona de ablación en la superficie del glaciar. En la Línea de Equilibrio Glaciar la acumulación es igual a la ablación, o sea que el balance de masa anual sobre esta línea es cero. La porción del glaciar ubicada por encima de esta línea tendrá al final del año un balance de masa positivo (zona de "acumulación»), mientras que la parte del glaciar ubicada por debajo de la ELA tendrá un balance de masa negativo (zona de "ablación»).

FIGURA 6: ZONAS DE UN GLACIAR

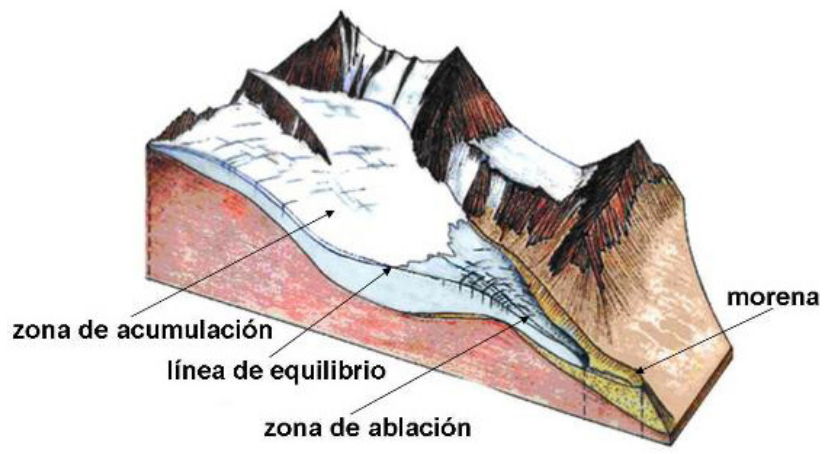

Fuente: Suárez, 2012

Para calcular la ELA se siguió el método indirecto, el cual se fundamenta en la cantidad anual de precipitación y la temperatura promedio anual. La ventaja de este método es que permite relacionar el clima con el comportamiento glaciar. Para calcular la ELA, se siguieron dos métodos:
1. La ecuación de Greene et al. (2002) establecida para las zonas tropicales:

$\mathrm{ELA}=537+1,01 \mathrm{FH}-0,51 \mathrm{PA}$

Donde FH es la altura de la isoterma $0^{\circ}$ y PA la precipitación anual en $\mathrm{mm}$.

2. La ecuación de Condom (2002) y Condom et al. (2007) establecida para una zona intermedia de los Andes que va desde $10^{\circ}$ de latitud norte hasta $55^{\circ}$ de latitud sur.

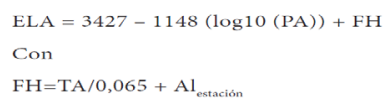

Donde TA es la temperatura promedio anual en grados centígrados y $\mathbf{A l}$ es la altitud de la estación de medición, el coeficiente de 0,065 es el gradiente altitudinal de temperatura.

Para calcular la ELA, fue necesario datos de entrada como temperatura y precipitación. Para estos parámetros se consideró los datos de las estaciones Yauricocha, Corihuarmi, Huichicocha, Cosmos y Telapacha ubicadas en los departamentos de Lima, Junín y Huancavelica, estos se ubican a una altitud entre 4500 a $4700 \mathrm{msnm}$. Bajo esta consideración se procedió a calcular la temperatura en donde la isoterma será $0^{\circ} \mathrm{C}$ para cada año dado que el gradiente de temperatura es de $0.65^{\circ} \mathrm{C}$ por cada 100 metros que se asciende en altitud. En el caso de la precipitación se ha hecho solo una aproximación referencial ya que es un parámetro más variable y dada la escases de la información a la fecha no se puede llegar a conclusiones al $100 \%$ valederas.

Como una fuente alternativa se ha incorporado información del Tropical Rainfall Measuring Mission - TRMM (https://pmm.nasa.gov/trmm). La información analizada se muestra en Cuadro 6.

De los dos métodos evaluados, se consideró al segundo método (zonas intermedias en los Andes) puesto que el área evaluada se ubica a una latitud aproximada $12^{\circ}$ sur, además este método es el que presenta un mayor grado de relación y ubicación de la ELA.

Como se observa, la Línea de Equilibrio Glaciar se ubica cada vez a altitudes mayores, así en 1990 se ubicaba a una altitud de $5134 \mathrm{msnm}$, para el año 1995 se ubicó a $5170 \mathrm{msnm}$, en el 2000 la ELA estaba a 5 206 msnm, mientras que en el 2005 se ubicó a una 
CUADRO 6: UBICACIÓN DE LAS ESTACIONES CONSIDERAS

\begin{tabular}{|c|c|c|c|c|c|c|c|}
\hline \multirow[t]{2}{*}{ Estación } & \multirow[t]{2}{*}{ Departamento } & \multicolumn{2}{|c|}{ Coordenadas } & \multirow{2}{*}{$\begin{array}{l}\text { Altitud } \\
\text { msnm }\end{array}$} & \multirow[t]{2}{*}{ Parámetro } & \multirow[t]{2}{*}{ registro } & \multirow[t]{2}{*}{ Fuente } \\
\hline & & Lat. & Long. & & & & \\
\hline Cosmos & Junín & $12^{\circ} 09^{\prime}$ & $75^{\circ} 34^{\prime}$ & 4575 & \multirow{5}{*}{$\begin{array}{l}\text { Precipitación } \\
\text { Temperatura }\end{array}$} & $1982-1989$ & \multirow{5}{*}{ SENAMHI } \\
\hline Yauricocha & Lima & $12^{\circ} 19^{\prime}$ & $75^{\circ} 43^{\prime}$ & 4675 & & $1990-2009$ & \\
\hline Telepacha & Huancavelica & $12^{\circ} 45^{\prime}$ & $75^{\circ} 18^{\prime}$ & 4400 & & $1973-1982$ & \\
\hline Corihuarmi & Junín & $12^{\circ} 34^{\prime}$ & $75^{\circ} 34^{\prime}$ & 4636 & & $\begin{array}{l}2006-2009 \\
2007-2009\end{array}$ & \\
\hline Huichicocha & Junín & $12^{\circ} 23^{\prime}$ & $75^{\circ} 31^{\prime}$ & 4700 & & $1965-2005$ & \\
\hline
\end{tabular}

Fuente: Elaboración propia

CUADRO 7: PRECIPITACIÓN TOTAL MENSUAL - ESTACIÓN YAURICOCHA

\begin{tabular}{|c|c|c|c|c|c|c|c|c|c|c|c|c|c|}
\hline Año & Ene & Feb & Mar & $A b r$ & May & Jun & Jul & Ago & Set & Oct & Nov & Dic & Total \\
\hline 1987 & 315.3 & 180.7 & 89.8 & 39.1 & 12 & 20.5 & 70.6 & 13.2 & 10.7 & 27.8 & 63.7 & 143.8 & 987.2 \\
\hline 1988 & 209.1 & 129.3 & 214.4 & 163.9 & 21.3 & 10.3 & 0 & 10.7 & 33.8 & 60.6 & 81.4 & 175.3 & 1110 \\
\hline 1989 & 196.8 & 176.7 & 212 & 49.3 & 17.7 & 27.6 & 8.5 & 49.7 & 61.4 & 86.2 & 38.4 & 41.8 & 966.1 \\
\hline 1990 & 148.9 & 90.9 & 107.3 & 68.6 & 51.1 & 32.7 & 4.5 & 19.4 & 54.7 & 121 & 124.2 & 183.4 & 1007 \\
\hline 1991 & 117.9 & 172.4 & 171.1 & 138 & 6.5 & 41.8 & 8.4 & 9.7 & 20.2 & 118.3 & 48.9 & 87.5 & 940.7 \\
\hline 1992 & 129.3 & 107.6 & 128.3 & 42.1 & 5.8 & 12.6 & 7.5 & 15.9 & 7.3 & 113.8 & 46.8 & 51.7 & 668.7 \\
\hline 1993 & 164.5 & 183.3 & 183 & 177.9 & 26.8 & 2.1 & 18.7 & 18.1 & 30.7 & 120.7 & 185.3 & 159.9 & 1271 \\
\hline 1994 & 228.2 & 210.7 & 225.6 & 163.6 & 40 & 8.3 & 7.4 & 17.4 & 54 & 44 & 36.9 & 91.1 & 1127 \\
\hline 1995 & 148.2 & 101.8 & 191.8 & 68.7 & 9.8 & 2.8 & 7.8 & 1.9 & 40.3 & 65.2 & 78.4 & 131.1 & 847.8 \\
\hline 1996 & 178.3 & 162.2 & 102.2 & 96.1 & 14.3 & 1.8 & 3.3 & 22.8 & 40.6 & 44.1 & 39.2 & 133.5 & 838.4 \\
\hline 1997 & 157.9 & 183.8 & 66.8 & 30.4 & 8.2 & 4 & 1 & 57.5 & 38.7 & 88.6 & 108.6 & 182.8 & 928.3 \\
\hline 1998 & 249.8 & 176.1 & 177 & 65.3 & 0.7 & 12.9 & 2 & 7.1 & 15.9 & 80.9 & 88.9 & 99.8 & 976.4 \\
\hline 1999 & 113.7 & 233.4 & 156.1 & 143.8 & 47.3 & 2.6 & 8.8 & 5.4 & 24.7 & 82.5 & 62.4 & 172 & 1053 \\
\hline 2000 & 191.7 & 194.9 & 227.6 & 63.1 & 53.8 & 2.7 & 19.2 & 29.1 & 23.9 & 131.7 & 65.4 & 188.2 & 1191 \\
\hline 2001 & 208.1 & 123 & 242.7 & & 30 & 4.1 & 21.4 & 10.2 & 47.1 & 71.4 & 109.7 & 46.5 & 914.2 \\
\hline 2002 & 82.7 & 211.7 & 240.5 & 73.5 & 36.9 & 6.1 & 32.8 & 16.8 & 57.4 & 98.3 & 148 & 134 & 1139 \\
\hline 2003 & 183.2 & 193.9 & 197.3 & 111.8 & 30.6 & 0 & 8.6 & 12.2 & & 139.2 & 65.9 & 163.8 & 1107 \\
\hline 2004 & 34.9 & 172.7 & 150 & 76.1 & 6.7 & 10.2 & 7.2 & 14.7 & 90.7 & 114.4 & 77.3 & 184 & 938.9 \\
\hline 2005 & 229.7 & 121 & 247.6 & 90 & 3.6 & 4.7 & 1.1 & 49 & 39.3 & 102.1 & 121.6 & 133 & 1143 \\
\hline 2007 & 247.6 & 138.2 & 225 & 102.1 & 31.1 & 5.1 & 0 & 0 & 30.1 & 84.7 & 50.8 & 120.3 & 1035 \\
\hline 2008 & 162.7 & 236.6 & 60 & 47.2 & 10.3 & 2.7 & 0 & 18 & 44.9 & 89.9 & 43 & 131.7 & 847 \\
\hline 2009 & 155.7 & 240 & 111.9 & 115.1 & 6.3 & 0 & 2.4 & 66.8 & 27.3 & 103.4 & 182.4 & 267.9 & 1279 \\
\hline
\end{tabular}

Fuente: SENAMHI

altitud de $5277 \mathrm{msnm}$, en tanto que en el 2010 se ubicó a 5310 msnm mientras que en el 2015 se ubicó a 5343 .

\section{5 Área de Acumulación Glaciar}

El área de Acumulación Glaciar (ARR) se determinó a partir de los datos de la ELA y usando los programas computacionales del SIG. Una vez identificada la altitud de la ELA, se obtuvo la proporción entre la superficie de la zona de acumulación y la superficie total del glaciar (en porcentaje), valor que se conoce como Área de Acumulación Glaciar.

Con ayuda de los programas Google Earth y el ArcGIS, se determinaron las lenguas glaciares de los nevados antes indicados, en conjunto se han identificado 8 glaciares que aportan sus aguas a la cuenca del rio Cańete. Para su mejor comprensión los 
CUADRO 8: TEMPERATURA MEDIA MENSUAL (TC) - ESTACIÓN YAURICOCHA

\begin{tabular}{|c|c|c|c|c|c|c|c|c|c|c|c|c|c|}
\hline Año & Ene & Feb & Mar & Abr & May & Jun & Jul & Ago & Sep & Oct & Nov & Dic & Anual \\
\hline 1999 & 13.8 & 13.3 & 14.7 & 14.9 & 15.6 & 16.2 & 14.7 & 16.4 & 16.5 & 15.3 & 17.2 & 15.9 & 15.4 \\
\hline 2000 & 14.8 & 14.4 & 13.9 & 13.7 & 15 & 15.5 & 15.2 & 14.8 & 16.9 & 15.4 & 18.5 & 16.1 & 15.4 \\
\hline 2001 & 13.9 & 14.6 & 14.6 & 14.6 & 15.2 & 14.3 & 14.6 & 14.6 & 15.6 & 16.0 & 16.2 & 16.3 & 15.0 \\
\hline 2002 & 16.9 & 15.3 & 15.0 & 15.4 & 15.3 & 15.2 & 13.9 & 14.5 & 14.8 & 15.6 & 14.9 & 15.8 & 15.2 \\
\hline 2003 & 15.9 & 15.8 & 14.5 & 14.9 & 14.8 & 15.8 & 15.0 & 15.0 & 15.2 & 17.3 & 17.8 & 15.4 & 15.6 \\
\hline 2004 & 17.2 & 15.0 & 15.4 & 16.2 & 17.0 & 13.9 & 14.3 & 13.6 & 14.0 & 15.1 & 16.3 & 14.3 & 15.2 \\
\hline 2005 & 16.0 & 15.7 & 15.3 & 15.9 & 16.7 & 16.7 & 16.1 & 16.2 & 15.5 & 15.7 & 17.1 & 15.2 & 16.0 \\
\hline 2006 & 14.8 & 14.8 & 14.5 & 15.3 & 16.0 & 14.6 & 15.0 & 15.1 & 16.2 & 15.8 & 14.9 & 15.7 & 15.2 \\
\hline 2007 & 15.8 & 15.8 & 14.6 & 15.0 & 16.0 & 15.4 & 15.3 & 15.9 & 14.6 & 15.9 & 16.7 & 15.7 & 15.6 \\
\hline 2008 & 14.3 & 14.1 & 14.1 & 15.4 & 15.9 & 15.8 & 15.7 & 16.1 & 16.1 & 15.7 & 16.5 & 16.0 & 15.5 \\
\hline 2009 & 14.5 & 14.1 & 14.2 & 14.9 & 15.5 & 16.2 & 15.0 & 15.9 & 16.4 & 16.6 & 16.1 & 15.4 & 15.4 \\
\hline 2010 & 15.3 & 15.5 & 15.6 & 16.5 & 17.1 & 16.1 & 17.6 & 16.8 & 16.5 & 16.4 & 16.6 & 15.1 & 16.3 \\
\hline
\end{tabular}

Fuente: SENAMHI

CUADRO 9: ELA SEGÚN LOS MÉTODOS DE GREENE ET AL. (ZONAS TROPICALES) Y CONDOM ET ÁL. (ZONAS INTERMEDIAS DE LOS ANDES).

\begin{tabular}{|c|c|c|}
\hline Año & $\begin{array}{c}\text { Zonas Tropicales } \\
(\mathrm{msnm})\end{array}$ & $\begin{array}{c}\text { Zonas Intermedias de } \\
\text { los andes }(\mathrm{msnm})\end{array}$ \\
\hline 1990 & 5225 & 5134 \\
\hline 1995 & 5265 & 5170 \\
\hline 2000 & 5232 & 5206 \\
\hline 2005 & 5307 & 5277 \\
\hline 2010 & 5339 & 5310 \\
\hline 2015 & 5384 & 5343 \\
\hline
\end{tabular}

Fuente: Elaboración propia

glaciares han sido identificados de la siguiente manera: AG-01, AG-02 hasta AG-08.

CUADRO 10: CÁLCULO DEL ÁREA RATIO ACUMULATION DE LOS GLACIARES

\begin{tabular}{|c|c|c|c|c|c|c|c|c|}
\hline \multirow{2}{*}{ Año } & \multicolumn{7}{|c|}{ AAR de los glaciares (\%) } \\
\cline { 2 - 9 } & AG-01 & AG-02 & AG-03 & AG-04 & AG-05 & AG-06 & AG-07 & AG-08 \\
\hline 1990 & 62.0 & 45.0 & 55.0 & 50.0 & 41.0 & 39.0 & 38.0 & 48.0 \\
\hline 1995 & 61.0 & 40.0 & 64.0 & 36.0 & 37.0 & 50.0 & 42.0 & 46.0 \\
\hline 2000 & 59.0 & 54.0 & 64.0 & 36.0 & 37.0 & 50.0 & 42.0 & 45.0 \\
\hline 2005 & 60.0 & 50.0 & 62.0 & 45.0 & 45.0 & 60.0 & 54.0 & 62.0 \\
\hline 2010 & 66.0 & 56.0 & 65.0 & 56.0 & 54.0 & 62.0 & 56.0 & 65.0 \\
\hline 2015 & 63.0 & 55.0 & 63.0 & 53.0 & 52.0 & 66.0 & 46.0 & 61.0 \\
\hline
\end{tabular}

Fuente: Elaboración Propia
FIGURA 7: COMPARATIVO ENTRE LOS MÉTODOS PARA CALCULAR EL ELA

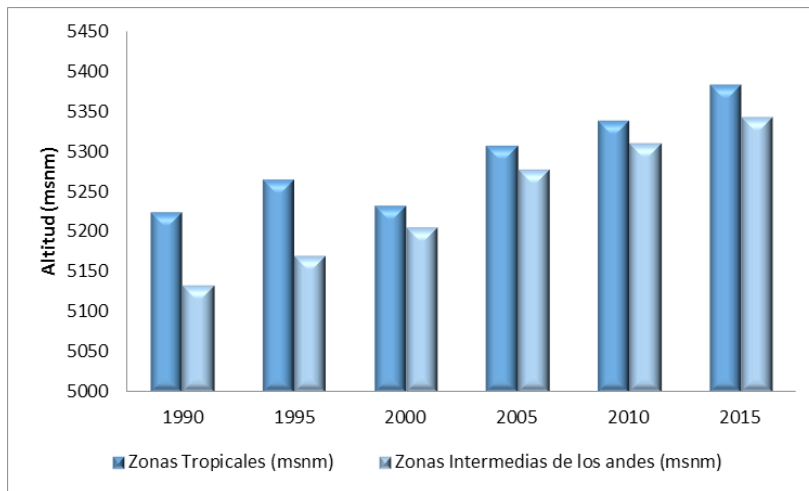

Fuente: Elaboración propia

Figura 8: EVOLUCIÓN DEL ÁREA DE ACUMULACIÓN DEL GLACIAR

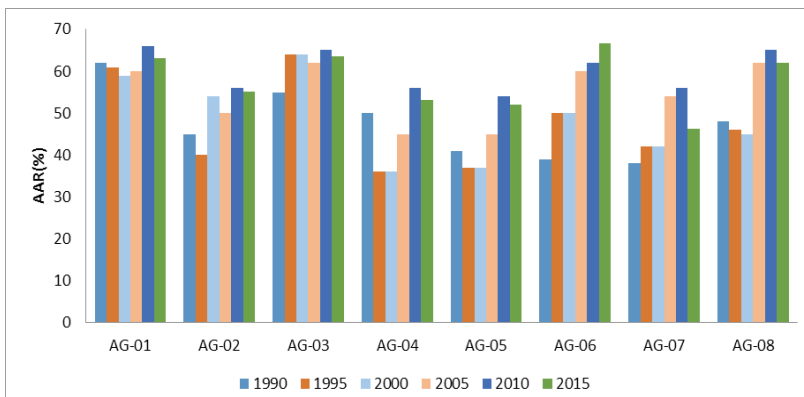

Fuente: Elaboración propia.

El ARR de los glaciares oscila entre 40 a 66\%, esto tal como se muestra en el Cuadro 10, la relación entre el área de la zona de acumulación y el área total es cada vez mayor en los ańos más recientes, esto 
sería el resultado entre otros factores del incremento constante de la temperatura que se está dando en las últimas décadas y que tiene un efecto dramático sobre los ecosistemas andinos.

\section{Conclusiones}

El cambio climático manifestado en una distorsión de las características climáticas en diferentes partes del mundo está generando graves consecuencias en los recursos hídricos, estos cambios son básicamente el incremento gradual de la temperatura, el que se está acelerando rápidamente en estos últimos años. Así los nevados se constituyen en los más sensibles a los cambios ante el incremento de la temperatura.

En 1990 el área de los nevados analizados fue de $68.9 \mathrm{~km}^{2}$, y para el año presento un área de $62.1 \mathrm{~km}^{2}$, en tanto que para el año 2000 su área fue de $57.34 \mathrm{~km}^{2}$, en el 2005 el área fue de $54.75 \mathrm{~km}^{2}$, en el 2010 el área era de $52.98 \mathrm{~km}^{2}$ y en el 2015 el área fue de $47.74 \mathrm{~km}^{2}$; en este periodo (1990-2015) los nevados presentaron una pérdida del 30\% de su área respecto año cero (1990).

Del análisis del retroceso glaciar desde 1990 hasta el 2015 (intervalo de 5 ańos) se estima que los nevados Ticlia, Llongote, Pariacaca y Collquepucro podrían desaparecer en el 2076.

El volumen total de los nevados en 1990 superaba los $15 \mathrm{~m}^{3}$, disminuyendo $2 \mathrm{~m}^{3}$. A partir de esta fecha ha ido perdiendo su volumen en un rango de 0.4 a $2 \mathrm{~m}^{3}$. Hasta que en el 2015 presentó un volumen de $9.22 \mathrm{~m}^{3}$.

Como es de observar la Línea de Equilibrio Glaciar se ubica cada vez a altitudes mayores, así en 1990 se ubicaba a una altitud de $5134 \mathrm{msnm}$, para el año 1995 se ubicó a $5170 \mathrm{msnm}$, en el 2000 el ELA estaba a 5 206 msnm, mientras que en el 2005 se ubicó a una altitud de $5277 \mathrm{msnm}$, en el 2010 se ubicó a 5310 msnm y finalmente en el 2015 se ubicó a 5343 msnm.

El área de acumulación glaciar sigue siendo importante, aunque estos se están reduciendo con el paso de los años, estas áreas oscilan entre 40 a 66\%.

\section{Referencias bibliográficas}

ANA (Autoridad Nacional del Agua). (2014). Inventario de glaciares del Perú. Lima: ANA.

ArizA, A. (2013). Productos LDCM - Landsat 8. Instituto Geográfico Agustín Codazzi. Bogotá.

Cogley, J. G. et al. (2011). Glossary of Glacier Mass Balance and Related Terms, UNESCO-IHP, Paris.

IGP (Instituto Geofísico del Perú). (2009). Evolución y fluctuaciones de los frentes glaciares del nevado Huaytapallana. Lima.

Kaser, G. y Georges, C. (1997). "Changes of the equilibrium-line altitude in the tropical Cordillera Blanca, Peru, 1930-1950, and their spatial variations». Annals of Glaciology, 24: 344-349. Austria. 